\title{
Transposition, apotheosis and benign metamorphosis-lymph node
}

Keywords: neoplastic tissue, tumour, proliferative lesions, lymph node, resembling structures, thyroid follicles

\section{Introduction}

Ectopic foci of non neoplastic tissue situated in the lymph node are designated as benign inclusions. A sequel to a metastatic process induces the epithelial cells in a lymph node which may be clinically significant on account of the probable impact in the determination of tumour staging and the employment of subsequent therapeutic options. Therefore, a distinction from the benign proliferative lesions in the lymph node (vascular proliferations, T lymphocyte clusters, hamartomas, viral infections or angiomyomas) is a pre-requisite. Intramural locations may be preferred and the inclusions may elucidate as epithelial cysts or duct resembling structures. The pathogenesis of benign lymphoid inclusions comprises of:

1. Transference of dissociated epithelium as a form of benign metastasis.

2. Evolving heterotopias.

3. Metaplasia of native multi-potent cells.

The cells may be derived from the paramesonephric ducts, salivary gland tissue, breast tissue, thyroid follicles, squamous epithelium or mesothelial incorporation. Paramesonephric duct like configurations and inclusions are preferentially located in pelvic lymph nodes and may simulate the uterine tube epithelium. Breast tissue inclusions are chiefly constituted of ectopic mammary glands and ducts of diverse morphology and unknown aetiology. Thyroid inclusions may be incorporated in the cervical and axillary lymph nodes. Mesothelial inclusions appear particularly in the mediastinal lymph nodes of the patients with pleural and pericardial effusions. The occurrence of melanocytic cells discovered in the lymph nodes are attributed to the faulty migration of neural crest cells or they may arise as benign metastasis of existing dermal nevi. The diagnosis of benign inclusions is necessitated in order to exclude adenocarcinomatous lymph node transformation and metastasis in patients presenting with benign nodal proliferations. The existence of benign inclusions was initially recounted by Reis et al. in $1897 .{ }^{1}$ These are defined as tubular spaces corresponding to cysts, appearing in lymph nodes of patients who may undergo surgical interventions for malignant tumours of the uterus, cervix and vulva. Inclusions may as well arise in non malignant disease processes and may be situated in locales extraneous to the pelvic cavity such as in the lumbar, mediastinal, parotid, submandibular, jugular, hepatic and iliac lymph nodes. Brooks et al divided the inclusions in three sub-categories, epithelial, nevomelanocytic and decidual. ${ }^{2}$

\section{Clinical exponents}

1. The benign epithelial inclusions may be discerned coincidentally and may typically lack specific symptoms. The diagnostic significance of the benign inclusions is considerable as they may be misinterpreted for metastatic malignant processes which may result in the employment of extensive and expensive investigative and therapeutic options (Table 1).
Volume 6 Issue 5 - 2018

\author{
Anubha Bajaj \\ Punjab University, India
}

Correspondence: Anubha Bajaj, Designation Consultant Pathologist, Punjab University, Chandigarh, India, Email anubha.bajaj@gmail.com, anubha.bajaj@yahoo.com

Received: June 18, 2018 | Published: September 24, 2018

Table I Selective sites of benign lymph node inclusions

\begin{tabular}{ll}
\hline Tissues favoring heterotopia & Implicated lymph nodes \\
\hline Mammary gland tissue & Axillary nodes \\
Aggregates of nevus cells & Axillary nodes \\
Blue nevus & Axillary nodes \\
Squamous Epithelium & Cervical \& peri-pancreatic nodes \\
Salivary Gland Tissue & Cervical nodes \\
Thyroid Follicles & Cervical nodes \\
Decidual Tissue & Pelvic nodes \\
Paramesonephric Epithelium & Pelvic nodes \\
Intestinal Glands & Mesenteric nodes \\
Mesothelial Cells & Mediastinal and Retroperitoneal \\
\hline
\end{tabular}

Adapted from: Pantanowitz \& Upton 2003. ${ }^{3}$

2. Inclusions of the lymph node situated in the intramural locations may be configured in cysts or as innumerable duct replicating formations. The epithelial cells articulating the inclusions may arise from the paramesonephric ducts, salivary glands, breast tissue, thyroid follicular epithelium, squamous epithelium and the mesothelium.

3. Lymph node inclusions of paramesonephricus sub-type may especially emerge in the pelvic lymph nodes and infrequently in the axillary lymph nodes. The concurrence with adenocarcinoma of the mammary glands is debatable.

4. Ectopic breast tissue is more prevalent in the sentinel lymph nodes, in contrast to adjunctive axillary nodes, on account of an obligatory embryologic connection.

5. Decidual inclusions are elucidated in the paramesonephric processes and may originate from submesothelial cells secondary to a hormonal trigger.

6. Lymph node inclusions of mammary gland origin may be composed of deformed mammary gland ducts with divergent morphology and a dual cell population comprising of luminal cuboidal /columnar epithelial cells and basal cells depicting myopithelial differentiation (Figure 1). 


\section{BREAST TISSUE INCLUSIONS -}

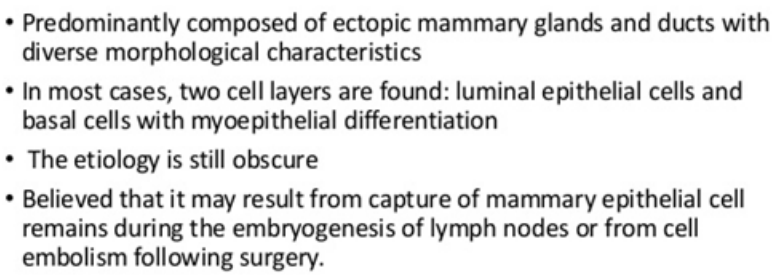

- Predominantly composed of ectopic mammary glands and ducts with diverse morphological characteristics

- In most cases, two cell layers are found: luminal epithelial cells and basal cells with myoepithelial differentiation

- The etiology is still obscure

- Believed that it may result from capture of mammary epithelial cell remains during the embryogenesis of lymph nodes or from cell embolism following surgery.

Figure I Origin of mammary inclusions.

7. Inclusions identical to thyroid glandular epithelial cells tend to localize in the cervical and axillary lymph nodes. Diagnostic paradigms for benign inclusions of the thyroid gland are i) The inclusion should be predominantly solitary, microscopic and situated in the subcapsular sinus of the lymph node. ii) Follicles should typically be symmetrical in size, may contain abundant colloid and may be lined by a uniformly aligned cuboidal epithelial cell layer. Anomalous patterns or microscopic features such as nuclear hyperchromasia, atypical mitosis, papillary formations or psammoma bodies should be nonexistent. iii) Essentially, the thyroid gland should be clinically normal, euthyroid and non palpable. These inclusions arise on account of the concordance of the embryologic tissues from which lymph node and thyroid glands arise.

8. Mesothelial inclusions may exclusively appear in the mediastinal lymph nodes draining pleural/ pericardial effusions. Contrary to the proliferating neoplastic cells, the mesothelial inclusions usually exist in the lymphatic sinus of the node and may be susceptible to a degenerative process. Melanocytic cells may accumulate in the nodal capsule as a consequence of faulty transmigration of the neural crest cells or with an emergence of a benign metastasis in definitive dermal nevi. Lymph node inclusions of the epithelial and nevomelanocytic category may be preponderant in the axillary lymph nodes. These lymph node inclusions are exhibited as being of glandular outline.

9. Stratified squamous epithelial cells may create cysts and duct like structures which may appear as lymph nodes inclusions and may be identical to the heterotopic tissue. Postulates of melanoma based lymph node inclusions are consistently elucidated as i) Abnormal and restricted migration from the embryonic neural crest as demonstrated in the existence of blue nevi at aberrant locations such as the prostate, cervix, vagina and uterus. ii) Nevus cells may transmigrate to the adult lymph node due to clefts in the lymphatic endothelium thereby indicating that benign metastasis from a cutaneous lesion may lack a malignant transformation. The lesions require a distinction from a metastatic malignant melanoma.

10. Cervical lymph nodes with inclusions of salivary glands may exhibit expansive, reactive lymphoid follicles with prominent germinal centres with numerous tingible body macrophages, foci of monocytoid cells, immunoblasts, plasma cells and vascular proliferation. Disseminated inclusions of salivary gland ducts and acini may not manifest lymphocytic permeation and destruction, as is exemplified in Sjogren's syndrome and Mikulicz's disease (Figure 2 - Figure 4$){ }^{3}$

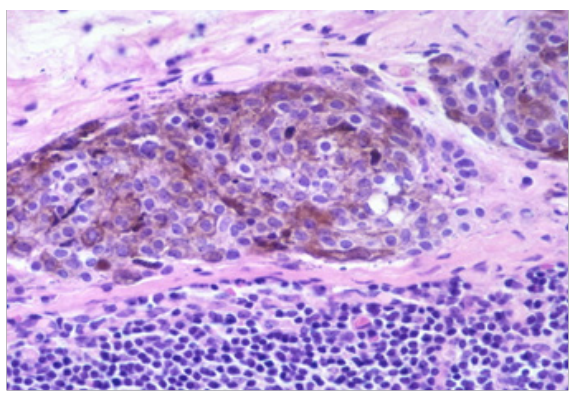

Figure 2 Nevus cell inclusions containing melanin.

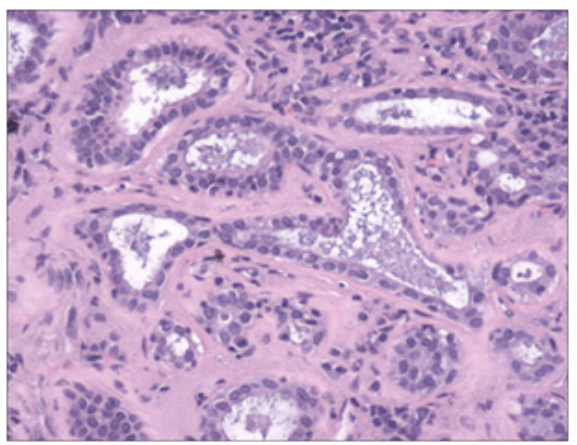

Figure 3 Benign mammary inclusions with glandular configuration.

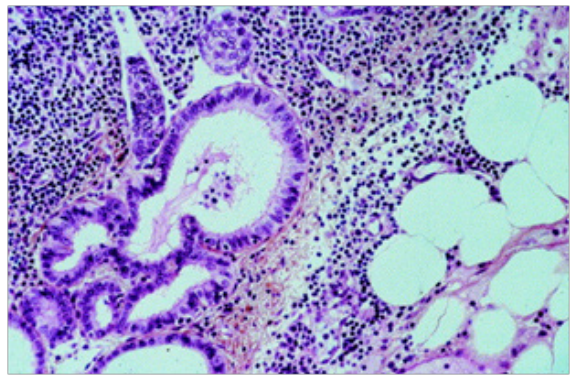

Figure 4 Cystic mullerian duct inclusions in the lymph node.

Categorization of lymph node inclusions: Lymph node inclusions may be categorized as:

1. Salivary glands inclusions: Inclusions from the salivary glands are frequent in the upper cervical lymph nodes and may manifest as a component of the developmental embryogenesis. Intact, undamaged glandular ducts and acini may be elucidated. The lymph node may manifest reactive follicles with prominent germinal centres with numerous tingible body macrophages, plasma cells and monocytoid cells. The concordant sjogren's $\&$ mikulicz syndrome may depict a lymphocytic infiltration of the ducts and acini. ${ }^{4}$ A malignant transformation may emerge with these inclusions, usually as a potential warthin's tumour, a pleomorphic or a monomorphic adenoma, a mucoepidermoid carcinoma or an acinic cell carcinoma.

2. Squamous epithelium: Cystic configurations inter-lined by a well differentiated squamous epithelium may ensue in the upper cervical lymph nodes. These may be identical to aberrant branchial pouch derivatives - thus designated as "benign lymphoepithelial cyst" and are surmised to appear due to cystic dilatation of pre-existent cysts. Peri-pancreatic lymph nodes 
may also display identical epithelium lined cystic inclusions. A demarcation from a metastatic well differentiated squamous cell carcinoma with superimposed cystic change may be mandated. ${ }^{5}$

3. Thyroid follicles Inclusions: Capsular or sub-capsular location of inclusions of non-pathogenic thyroid tissue in the midcervical lymph nodes may be elucidated in concordance with a normal thyroid gland. ${ }^{5}$ Distinctive colloid follicles lined by a low cuboidal epithelium lacking atypia may be exemplified. Configurations such as papillae or psammoma bodies may be absent. Follicles may be detected in the peripheral sinus. ${ }^{4}$

4. Decidual reaction: Pelvic lymph nodes usually delineate a decidual reaction which may simulate a malignant metastasis. It may occur in the stromal cells of co-existent conditions such as the Endometriosis. The adjunctive stromal cells or the hormonally susceptive cells are implicated in the genesis of the anomaly. ${ }^{5}$

5. Mullerian type epithelium: Glandular inclusions interlined by cuboidal cells simulating a mullerian or coelomic epithelium may be located in the pelvic lymph nodes. The inclusions may resemble the peritoneal lesions denominated as "endosalpingosis". Metastasis from a low grade ovarian neoplasm which may extend in the peripheral sinus, lesions which form papillae, the emergence of psammoma bodies or cellular proliferations configuring small, laminated layers are the conditions which require a distinction from the inclusions of mullerian type epithelium. Nodal endometriosis may accompany the glandular inclusions with an encompassing endometrial type stroma. $^{5}$

6. Nevus cells: Nevus inclusions may articulate as a sinus, trabaculae, nests, cords, linear strands or may be incorporated in the capsule. The cells are monomorphic with an indistinct border, pink cytoplasm, round to oval nuclei, indistinct nucleoli and contain granules of melanin. Sporadic cell clusters may be up-to $2.1 \mathrm{~mm}$ in magnitude. Mitosis is usually absent, in contrast to a malignant melanoma which may display metastasis in the marginal sinus. The nevus inclusions may be immune reactive for S 100 protein, tyrosinase, melanin and non reactive for immune markers such as melan A, HMB45 and Ki67. In contrast, the malignant melanoma appears reactive for S100 protein, melan A, HMB45 and Ki67. On ultra-structural examination, the nevus inclusions display uniform, round cells with random aggregates of cytoplasmic fibrils, mature melanosomes and dispersed nuclear chromatin. ${ }^{4}$ The axillary, cervical or inguinal lymph nodes may be implicated, however conventional nevus cells may be also be enunciated in the capsule of axillary lymph nodes, in the absence of an involved parenchyma. ${ }^{5}$

7. Mesothelial cells: Lymph nodes may infrequently delineate mesothelial cell aggregates accompanied by an absence of a malignant mesothelioma, from which a demarcation is required. ${ }^{5}$ Mesothelial windows may also be depicted as mediastinal cysts, situated in the sub-capsular spaces. The cellular configuration is that of a uniform tall, columnar epithelium with a low nucleocytoplasmic ratio, a distinct basement membrane and an absence of mitosis. The cells hypothetically arise from the pleural mesothelium. ${ }^{4}$

8. Mammary Inclusions: Axillary lymph nodes may frequently depict the ectopic mammary tissue. Inclusions of the breast tissue are demonstrated as mammary ducts in the subcapsular region. The cellular components described are the epithelium, myoepithelium and apocrine cells. Cystic spaces lined with low, uniform cuboidal epithelium lacking mitosis, hyperplasia or hyperchromasia may be evidenced. ${ }^{4}$ A singular layer of cuboidal epithelium interlining the tubules (hobnail appearance) or epithelial inclusions situated within or beneath the lymph node capsule may also be elucidated. A distinction is required from a metastatic breast carcinoma. The inclusions may delineate three categories i) glandular structures only ii) squamous cysts only iii) a combination of glandular and squamous epithelium. ${ }^{5}$

To surmise, an extensive morphological evaluation may be mandated for a substantial and definitive prognostic delineation of the benign lymph node inclusions as an immunohistochemical or biomolecular investigation may not categorically define a non-malignant tissue metamorphosis or the transformation into a neoplasm.

\section{Acknowledgements}

None.

\section{Conflict of interest}

The author declares that there is none of the conflicts.

\section{References}

1. Julia Regazzini Spinardi, Ísis Rocha Dias Gonçalves, Thiago Souza La Falc, et al. Benign Inclusions in Lymph nodes. Int $J$ Morphol. 2007;25(3):625-629.

2. Brooks JS, Li Volsi VA, Pietra GG, et al. Mesothelial cell Inclusions in Mediastinal Lymph Nodes mimicking Metastatic Carcinoma. AM J Clin Pathol. 1990;93(6):741-748.

3. Pantanowitz I, Upton M. Benign Axillary Lymph Node Inclusions. Breast J. 2003;9(1):56-57.

4. Harry L Ioachim, L Jeffrey Medeiros. Ioachim's Lymph Node Pathology. Fourth ed. 2009.

5. Juan Rosai. Rosai and Ackerman Surgical Pathology. Tenth ed. 2011. $2982 \mathrm{p}$.

6. Image 1 Courtesy: Breast Inclusions Chart: Slide share.

7. Image 2 Courtesy: Web-pathology.

8. Image 3 Courtesy: Pathology.jhu.edu.

9. Image 4 courtesy: Science direct. 